\title{
Non-invasive application for domestic pipeline monitoring and corrosion detection
}

\begin{abstract}
In this paper a method of assessment and control of condition for domestic pipelines is proposed and been tested for a pair. Most of the previous works has been designed for long range pipes as they may carry valuable liquids. But having a system to monitor domesticô pipes also can help to save so much time and energy in future as leaking itself can be destructive in structures. This system is applying much lower frequency (40 kHz) in compare to other similar systems (above hundreds megahertz) which leads to lower power consumption. This ultrasonic wave is applied to short distance pipes to observe the changes and detect the leakage base on adjusted thresholds amplitude. It is able to detect smallest changes as the matter of difference in acoustic waves for healthy and damaged pipes. The alarming system works by analyzing output signals from receivers. Ultrasonic sensors are having $40 \mathrm{kHz}$ central frequency for both transmitter and receiver with best responsibility at face to face condition. Acoustic sensors are working independently in pair and by dismissing one pair others will not affect continuity of data acquisition. For this paper a couple of transducer (a transmitter and receiver) operation has been investigated. By understanding the functionality of a pair can analyse show they are working in more numbers. This system is a cheap, easy to assemble, install and maintain for homes and most of public facilities such as schools, hospitals, parks, offices and business buildings. It doesnâ need any particular constriction or changes in pipeline structures.
\end{abstract}

Keyword: Ultrasonic waves; Threshold adjustment; Acoustic sensors; Continually data acquisition 